\title{
Impacto de la técnica de Cusp-Overlap en el requerimiento de marcapasos luego de un implante valvular aórtico percutáneo
}

\author{
Impact of Cusp-Overlap Technique on Pacemaker Requirement after Transcatheter Aortic \\ Valve Implantation
}

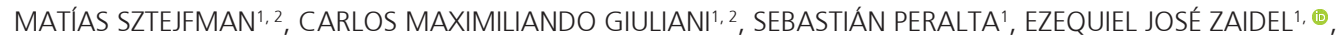
LUIS CARLOS SZTEJFMAN ${ }^{1,2}$, MARCELO OMAR BETTINOTTI'

\begin{abstract}
RESUMEN
Introducción: Las bradiarritmias persistentes que requieren el implante de un marcapasos definitivo son una complicación frecuente tras el implante valvular aórtico percutáneo (IVAP), pero un implante alto con técnica Cusp-Overlap podría evitar las alteraciones del sistema de conducción.

Objetivo: El objetivo fue determinar la tasa de uso de marcapasos en pacientes que recibieron IVAP con la técnica convencional coplanar en comparación con Cusp-Overlap.

Material y métodos: Entre 2017 y 2019 se analizaron 65 pacientes consecutivos de dos centros, que recibieron válvulas Evolut$R$ o Evolut-Pro: 50 implante coplanar y 15 Cusp-Overlap.

Resultados: La edad promedio era 80 años y no hubo diferencias en el riesgo por puntaje EuroSCORE. Los procedimientos con la técnica de Cusp-Overlap presentaron 0\% de requerimiento de marcapasos en comparación con 24,9\% en el implante convencional ( $\mathrm{p}=0,041)$. También hubo menos complicaciones mayores $(6,67 \%$ vs $42 \%$; OR = 0,09; IC 95\% 0,01-0,8; $\mathrm{p}=0,011)$. Conclusiones: En esta cohorte el IVAP con la técnica de Cusp-Overlap se asoció con una necesidad de marcapasos nula. Dado el potencial impacto a gran escala se necesita validar externamente los resultados obtenidos.
\end{abstract}

Palabras clave: Reemplazo de la válvula aórtica transcatéter - Bloqueo atrioventricular - Marcapasos artificial - Implantación de prótesis de válvulas cardíacas, métodos

\begin{abstract}
Background: Persistent bradyarrhythmias requiring permanent pacemaker implantation are a common complication after transcatheter aortic valve implantation (TAVI), but high implantation with cusp-overlap technique could prevent conduction system disturbances.

Objective: The aim of this study was to assess the rate of pacemaker use in patients who received TAVI with conventional coplanar technique compared with cusp-overlap technique.

Methods: A total of 65 consecutive patients from two centers receiving Evolut-R or Evolut-Pro valve implantation, 50 coplanar and 15 cusp-overlap, were analyzed between 2017 and 2019.

Results: Mean age was 80 years, and there were no differences in risk according to the EuroSCORE. The rate of pacemaker requirement was $0 \%$ in cusp-overlap procedures compared with $24.9 \%$ in those with conventional implantation; $\mathrm{p}=0.041$ ). In addition, cusp-overlap implants presented lower rate of major complications $(6.67 \%$ vs. $42 \%$; OR=0.09; $95 \%$ CI $0.01-0.8$; $\mathrm{p}=0.011$ ).

Conclusions: In this cohort, TAVI procedure with cusp-overlap technique was associated with null pacemaker requirement. Given the potential large-scale impact, external validation of results is needed.
\end{abstract}

Key words: Transcatheter aortic valve replacement - Atrioventricular block - Pacemaker, Artificial - Heart Valve Prosthesis Implantation/methods

\section{INTRODUCCIÓN}

El implante valvular aórtico percutáneo (IVAP) se ha establecido como el tratamiento estándar para la estenosis aórtica (EA) y su uso se extiende desde los casos inoperables hasta los de bajo riesgo. (1) Aunque se ha observado una reducción significativa en la morbilidad y mortalidad periprocedimiento algunas complicaciones siguen siendo frecuentes.

El bloqueo aurículo ventricular (BAV) que ocurre luego del IVAP es determinante de implantes de marcapasos permanentes (MCP), que oscilan entre el $5 \%$ y el $50 \%$ en

Rev Argent CARDiol 2021;89:140-144.http://dx.doi.org/10.7775/rac.es.v89.i2.19091

Recibido: 10/11/2020 - Aceptado: 18/01/2021

Dirección para separatas: Dr. Ezequiel Zaidel - Acuña de Figueroa 1224, (C1180AAX). Sanatorio Güemes. Departamento de Cardiología intervencionista - E-mail: ezaidel@fsg.edu.ar - Tel/Fax +54 1149598200

${ }^{1}$ Servicio de Cardiología intervencionista, Sanatorio Güemes, Buenos Aires, Argentina.

${ }^{2}$ Servicio de Cardiología intervencionista, Sanatorio Finochietto, Buenos Aires, Argentina. 
diferentes series. (2) Distintas determinantes del BAV luego del IVAP fueron agrupados por diferentes grupos en alteraciones eléctricas previas, calcificación, tipo de válvulas, e implante bajo de la válvula. $(3,4)$

A diferencia de la técnica convencional radiológica para el IVAP, con posición del radioscopio en oblicua anterior izquierda e implante coplanar, Tang sugirió utilizar la vista de fluoroscopia de "superposición de cúspides", Cusp-Overlap, para guiar el implante de válvulas autoexpandibles. (5) Con esa técnica el catéter se encuentra más centrado a través de la válvula aórtica, y una vista frontal de la cúspide no coronariana permite un implante más alto de la válvula con menor riesgo de embolización del dispositivo y de contacto con el sistema de conducción.

El objetivo de este trabajo fue determinar diferencias en la tasa de implante de marcapasos permanentes entre pacientes que recibieron IVAP con la técnica convencional o con la técnica Cusp-Overlap. La hipótesis fue que esta técnica se asociaría con menor tasa de implante de marcapasos.

\section{MATERIAL Y MÉTODOS}

Se realizó un análisis retrospectivo de una base de datos continua donde se registraron todos los procedimientos de IVAP en dos centros de alta complejidad de la ciudad de Buenos Aires (Argentina) entre enero de 2017 y diciembre de 2019. El seguimiento fue realizado durante la hospitalización, y a 30 días; dos autores recopilaron la información de cada procedimiento en forma prospectiva y la incorporaron a una base de datos.

El equipo de intervencionistas inició los IVAP en el año 2009, pero se seleccionó la fecha de enero 2017, a partir de la cual todos los pacientes recibieron válvulas autoexpandibles Evolut-R o Evolut Pro. Así, se superó la curva de aprendizaje, y se utilizaron válvulas de nueva generación en todos los casos. El 100\% de los casos fue realizado por acceso transfemoral.

Todos los pacientes sometidos a IVAP fueron considerados elegibles para este estudio; sin embargo, se excluyeron los pacientes con implante de marcapasos previo para el análisis del criterio de valoración principal, así como los pacientes con bloqueo de rama izquierda para el análisis de nuevos bloqueos de rama a 30 días. El seguimiento fue realizado utilizando información de la historia clínica y también mediante llamados telefónicos.

El criterio de valoración principal fue la necesidad de implante de un MCP definitivo durante la hospitalización y en el seguimiento a 30 días. Otros criterios de valoración fueron la incidencia de bloqueo completo de rama izquierda a 30 días y el criterio combinado de complicaciones mayores (implante de MCP, fuga paravalvular en grado más que leve, obstrucción aguda de una arteria coronaria, shock, sangrados mayores, muerte). La definición y la asignación de las variables fueron realizadas según la clasificación VARC-2; (6) se obtuvo la información de los registros del procedimiento, exámenes complementarios, y de las historias clínicas.

Al tratarse de un registro continuo de pacientes de dos instituciones, se evitaron sesgos de exclusión o de adjudicación mediante la revisión de la información por los investigadores Giuliani y Peralta.

\section{Análisis estadístico}

El tamaño muestral fue limitado por el intervalo de tiempo del estudio y el número de sujetos que recibieron IVAP en ambos hospitales. Las variables cualitativas se describieron como números y porcentajes. Las variables cuantitativas se describieron como media (desvío estándar) o mediana (intervalo intercuartilo) de acuerdo con su tipo de distribución. Para definir la normalidad de la distribución de los resultados se utilizó el método de Bartlett. Las comparaciones se realizaron utilizando análisis estadísticos convencionales $\left(\mathrm{chi}^{2}\right.$, prueba exacta de Fisher, $t$ de Student, análisis de varianza, de acuerdo al tipo de distribución de las variables). En ocasión de ausencia de información en alguna variable, se excluyó el caso del análisis; no se realizaron imputaciones. No hubo pérdidas en el seguimiento. Para los análisis de nuevos marcapasos se excluyeron los pacientes con marcapasos previos. Se utilizó el programa estadístico EpiInfo V7.0 ${ }^{\circledR}$ y se consideró significativo un valor de $\mathrm{p}<0,05$.

\section{Consideraciones éticas}

Los procedimientos de este estudio se realizaron acorde con los lineamientos de la declaración de Helsinki. El grupo de autores del análisis no tuvo acceso a los datos confidenciales de los sujetos.

\section{RESULTADOS}

Un total de 65 pacientes recibieron IVAP entre 2017 y 2019 en ambos centros con válvulas autoexpandibles Evolut- $R$ o Evolut-Pro. De ellos, 50 recibieron un implante convencional coplanar, mientras que a partir de noviembre 2019 se utilizó el implante alto con la técnica Cusp-Overlap en todos los casos (15 sujetos).

Se trató de una población con edad media de 80 años; la proporción de mujeres en el grupo Cusp-Overlap fue mayor (66\% vs $33 \%$ en coplanar, $\mathrm{p}<0,01)$. No se hallaron diferencias en el riesgo de los pacientes estratificado por el puntaje de la Sociedad de Cirujanos Torácicos (STS): $5,75$ (RIC 4-8,4) y 6,8 (5-8,9) respectivamente ( $\mathrm{p}=\mathrm{NS})$, sin embargo, el 85\% de ambos grupos presentaban síntomas de insuficiencia cardíaca en clase III o IV (Tabla 1).

La incidencia de bloqueo de rama izquierda transitorio a 30 días fue de 21,4\% en Cusp Overlap y $17 \%$ en el grupo convencional ( $\mathrm{p}=\mathrm{NS}$ ), con un solo caso de bloqueo de rama izquierda definitivo en Cusp Overlap (7,14\% vs $0 \%, \mathrm{p}=0,08)$. No se hallaron diferencias en el tiempo de fluoroscopia o el volumen de contraste utilizado, ni en la mortalidad (Tabla 2).

Luego de excluir los casos que presentaban marcapasos previo, (9 sujetos del grupo convencional y 1 caso del grupo Cusp-Overlap), se pudo determinar una nula tasa de implante de marcapasos en el grupo Cusp-Overlap en comparación con $24,9 \%$ (10 sujetos) en el grupo de implante convencional. El criterio de valoración combinado a 30 días también fue menor en el grupo Cusp-Overlap (7,14\% vs $43,9 \%$; OR $=0,09 ;$ IC 95\%: 0,01-0,82; $\mathrm{p}=0,001$ ) (Tabla 3).

\section{DISCUSIÓN}

El principal hallazgo de este estudio fue la nula tasa de requerimiento de marcapasos sin que ello implique un mayor riesgo de otras complicaciones para los pacientes sometidos a IVAP.

Las complicaciones posteriores al IVAP, como los BAV, no solo modifican el pronóstico de los pacientes, sino que 


\begin{tabular}{|c|c|c|c|}
\hline & CUSP OVERLAP $(\mathrm{n}=15)$ & CONVENCIONAL $(n=50)$ & $\mathbf{P}$ \\
\hline Proporción de pacientes & $23,08 \%$ & $76,92 \%$ & \\
\hline Varones & $33 \%$ & $66 \%$ & 0,02 \\
\hline Mujeres & $66 \%$ & $34 \%$ & \\
\hline Edad ( \pm DE) & $80,07(6,31)$ & $79,46(6,80)$ & 0,76 \\
\hline EuroScore II ( $\pm \mathrm{DE})$ & $6,32(4,66)$ & $8,95(7,68)$ & 0,19 \\
\hline STS (RIC) & $5,75(4-8,4)$ & $6,8(5-8,9)$ & 0,32 \\
\hline CF NYHA III-IV & $87 \%$ & $84 \%$ & 0,25 \\
\hline EPOC & $33,3 \%$ & $32 \%$ & 0,92 \\
\hline Enfermedad coronaria & $53,3 \%$ & $58 \%$ & 0,74 \\
\hline Angioplastia & $33,3 \%$ & $42 \%$ & 0,54 \\
\hline CRM & $13,3 \%$ & $18 \%$ & 0,67 \\
\hline Vasculopatía periférica & $6,67 \%$ & $28 \%$ & 0,08 \\
\hline ACV & $13,3 \%$ & $8 \%$ & 0,61 \\
\hline Fibrilación auricular & $13,3 \%$ & $16 \%$ & 0,80 \\
\hline Marcapasos previo & $6,67 \%$ & $16 \%$ & 0,67 \\
\hline Bloqueo de rama derecha & $0 \%$ & $14 \%$ & 0,18 \\
\hline Bloqueo de rama izquierda & $6,67 \%$ & $20 \%$ & 0,43 \\
\hline $\mathrm{FEY}( \pm \mathrm{DE})$ & 59 (187) & $55,3(13,9)$ & 0,40 \\
\hline Gradiente máximo mmHg ( $\pm \mathrm{DE})$ & $76,8(27,6)$ & $70,4(25,5)$ & 0,41 \\
\hline Gradiente medio $\mathrm{cm}^{2}( \pm \mathrm{DE})$ & $52,5(18,2)$ & $42,3(16,3)$ & 0,046 \\
\hline Área valvular aórtica mm (RIC) & $0,60(0,5-0,8)$ & $0,79(0,68-0,8)$ & 0,03 \\
\hline Anillo mínimo por TAC mm ( $\pm \mathrm{DE})$ & $20,4(2,7)$ & $22,4(2,4)$ & 0,018 \\
\hline Anillo máximo por TAC mm ( $\pm \mathrm{DE})$ & $26(3,4)$ & $26(3,4)$ & 0,99 \\
\hline Área del anillo por TAC $( \pm \mathrm{DE})$ & $434(107)$ & $488(86)$ & 0,07 \\
\hline Bicúspide & $0 \%$ & $4,4 \%$ & 0,40 \\
\hline Porcelana & $13,3 \%$ & $6,3 \%$ & 0,58 \\
\hline
\end{tabular}

ACV = accidente cerebrovascular; CF NYHA = clase funcional de la New York Heart Association; CRM = cirugía de revascularización miocárdica; DE = desviación estándar; $\mathrm{EPOC}=$ enfermedad pulmonar obstructiva crónica; FEY = fracción de eyección del ventrículo izquierdo; RIC = rango intercuartilos; STS = clasificación de la sociedad de cirujanos torácicos de Estados Unidos; TAC = tomografía axial computada

conllevan consecuencias económicas relevantes, dado que representan el $25 \%$ del costo del procedimiento. (7) En ese sentido, con las válvulas autoexpandibles utilizadas en esta cohorte, la tasa de bloqueos fue menor que la reportada en las series iniciales de IVAP (24,9\%), pero utilizando la técnica de Cusp-Overlap pasó a ser 0\%, que podría modificar -si son validados estos resultados en otras cohortes- los análisis de costo-efectividad de IVAP, principalmente en los países de recursos económicos limitados.

Si bien se han descrito como potenciales riesgos de la técnica la oclusión de los senos coronarios y las fugas paravalvulares, en esta serie no se observaron esas complicaciones. Por otro lado, el tiempo de fluoroscopia y el volumen de contraste yodado utilizado tampoco fueron mayores con esta nueva técnica; posiblemente se deba a una apropiada planificación previa previa al ingreso del paciente a la sala de procedimientos. (8) En este sentido, en esta serie el uso de tomografía previa con mediciones de anillo, senos coronarios, tracto de salida del ventrículo izquierdo, altura coronaria y planificación de la proyección en vista oblicua caudal derecha, probablemente hayan contribuido los resultados.

La ampliación del IVAP en los últimos ensayos clínicos hacia pacientes de menor riesgo hace que las complicaciones posteriores al IVAP cobren relevancia; por esa razón, consideramos que la correcta planificación, la elección de la prótesis, el acceso y la evaluación del riesgo de complicaciones eléctricas son fundamentales en la actualidad.

Como limitación, encontramos que el bajo número de pacientes no permitió realizar análisis de subgrupos. Dado que el mismo equipo médico realizó todos los procedimientos superando la curva de aprendizaje (más de 10 años desde el primer implante), los resultados pueden no extrapolarse a centros con menos experiencia. Finalmente, la determinación de implante de MCP fue hasta el día 30. Sin embargo, la tasa de nuevos BAV es menor del 3,3\% más allá del día 30 (9) con estas válvulas, en comparación con otras que presentan riesgo de bloqueos tardíos. (10) Destacamos también como limitación que el bajo número de pacientes no permitió realizar un análisis de sensibilidad. 


\begin{tabular}{|c|c|c|c|}
\hline & CUSP OVERLAP $(\mathrm{n}=15)$ & CONVENCIONAL $(n=50)$ & $\mathbf{p}$ \\
\hline Acceso transfemoral & $100 \%$ & $100 \%$ & 0,23 \\
\hline Anestesia total & $0 \%$ & $26 \%$ & 0,028 \\
\hline Conversión a cirugía abierta & $0 \%$ & $0 \%$ & 1 \\
\hline Gradiente máximo post, mmHg ( $\pm \mathrm{DE})$ & $10,5(4,2)$ & $12,4(6,7)$ & 0,30 \\
\hline Gradiente medio post, $\mathrm{mmHg}( \pm \mathrm{DE})$ & $5,7(2,1)$ & $5,9(3,4)$ & 0,81 \\
\hline Fuga paravalvular grave & $0 \%$ & $2 \%$ & 0,079 \\
\hline Fuga paravalvular moderada & $0 \%$ & $22 \%$ & 0,079 \\
\hline Sangrado mayor & $0 \%$ & $2 \%$ & 1 \\
\hline Complicación vascular mayor & $0 \%$ & $4 \%$ & 1 \\
\hline ACV o AIT & $0 \%$ & $2 \%$ & 1 \\
\hline ACV mayor & $0 \%$ & $0 \%$ & 1 \\
\hline IRA & $0 \%$ & $4 \%$ & 1 \\
\hline Tiempo de fluoroscopia (mins) & $12,06(1,1)$ & $12,34(2,8)$ & 0,72 \\
\hline Volumen de contraste (ml) & $91(12,5)$ & $99,5(24)$ & 0,19 \\
\hline Muerte hospitalaria & $0 \%$ & $0 \%$ & 1 \\
\hline Fuga paravalvular M-S 30 días & $0 \%$ & $28.5 \%$ & 0,07 \\
\hline ACV 30 días & $0 \%$ & $0 \%$ & 1 \\
\hline Muerte 30 días & $0 \%$ & $2 \%$ & 1 \\
\hline Nuevo MCP & $0 \%$ & $20 \%$ & 0,056 \\
\hline Criterio de valoración combinado & $6,67 \%$ & $42 \%$ & 0,011 \\
\hline
\end{tabular}

$\mathrm{ACV}=$ accidente cerebrovascular; $\mathrm{AIT}=$ accidente isquémico transitorio; $\mathrm{DE}$ = desvación estándar; $\mathrm{MCP}=$ marcapasos permanente; $\mathrm{M}-\mathrm{S}=$ moderada a severa; $\mathrm{ml}=$ mililitros

Tabla 3. Desenlaces luego de excluir pacientes con implante previo de marcapasos artificiales (9 casos en grupo convencional, 1 en grupo Cusp-Overlap)

\begin{tabular}{|lccc|}
\hline & CUSP OVERLAP $(\mathbf{n}=\mathbf{1 4})$ & CONVENCIONAL $(\mathbf{n}=\mathbf{4 1})$ & P \\
\hline Nuevo MCP & $0,14(0 \%)$ & $10 / 41(2, .9 \%)$ & 0,041 \\
\hline Nuevo BCRI transitorio & $3 / 14(21,4 \%)$ & $7 / 41(17 \%)$ & 0,70 \\
\hline Nuevo BCRI definitivo & $1 / 14(7,14 \%)$ & $0 \%$ & 0,08 \\
\hline EP combinado & $1 / 14(7,14 \%)$ & $18 / 41(43,9 \%)$ & 0,001
\end{tabular}

$\mathrm{BCRI}=$ bloqueo completo de rama izquierda del haz de His; $\mathrm{EP}=$ endpoint, criterio de valoración; $\mathrm{MCP}=$ marcapasos permanente

\section{CONCLUSIONES}

En esta serie de pacientes el IVAP utilizando la técnica de Cusp-Overlap fue seguro, y se asoció con una nula tasa de implante de MCP en el seguimiento a 30 días. Estos resultados deberían ser validados externamente dado el potencial beneficio de evitar un implante de marcapasos luego del IVAP.

\section{Declaración de conflicto de intereses}

Los autores declaran que no poseen conflicto de intereses.

(Véase formulario de conflicto de intereses de los autores en la web / Material suplementario).

\section{Fuentes de financiamiento}

No se recibieron financiamientos. Este artículo se realizó siguiendo las recomendaciones STROBE (11).

\section{BIBLIOGRAFÍA}

1. Baumgartner H, Falk V, Bax JJ, De Bonis M, Hamm C, Holm PJ, et al. 2017 ESC/EACTS Guidelines for the management of valvular heart disease. Eur Heart J 2017;38:2739-91. http://dx.doi.org/10.1093/ eurheartj/ehx391

2. Auffret V, Puri R, Urena M, Chamandi C, Rodríguez-Gabella T, Philippon F, Rodés-Cabau J. Conduction Disturbances After Transcatheter Aortic Valve Replacement: Current Status and Future Perspectives. Circulation 2017;136:1049-69. http://dx.doi.org/10.1161/ CIRCULATIONAHA.117.028352

3. van Rosendael PJ, Delgado V, Bax JJ. Pacemaker implantation rate after transcatheter aortic valve implantation with early and new-generation devices: a systematic review. Eur Heart J 2018;39:2003-13. http://dx.doi.org/10.1093/eurheartj/ehx785

4. Young Lee M, Chilakamarri Yeshwant S, Chava S, Lawrence Lustgarten D. Mechanisms of Heart Block after Transcatheter Aortic Valve Replacement - Cardiac Anatomy, Clinical Predictors and Mechanical 
Factors that Contribute to Permanent Pacemaker Implantation. Arrhythm Electrophysiol Rev 2015;4:81-5. http://dx.doi.org/10.15420/ aer.2015.04.02.81.

5. Tang GHL, Zaid S, Michev I, Ahmad H, Kaple R, Undemir C, et al. "Cusp-Overlap" View Simplifies Fluoroscopy-Guided Implantation of Self-Expanding Valve in Transcatheter Aortic Valve Replacement. JACC Cardiovasc Interv 2018;11:1663-5. http://dx.doi.org/10.1016/j. jcin.2018.03.018

6. Kappetein AP, Head SJ, Généreux P, Piazza N, van Mieghem NM, Blackstone EH, et al. Updated standardized endpoint definitions for transcatheter aortic valve implantation: the Valve Academic Research Consortium-2 consensus document. Eur Heart J 2012;33:2403-18. http://dx.doi.org/10.1093/eurheartj/ehs255.

7. Arnold SV, Lei Y, Reynolds MR, Magnuson EA, Suri RM, Tuzcu EM, et al. Costs of periprocedural complications in patients treated with transcatheter aortic valve replacement: results from the Placement of Aortic Transcatheter Valve trial. Circ Cardiovasc Interv 2014;7:829-36. http://dx.doi.org/10.1161/CIRCINTERVENTIONS.114.001395.

8. Francone M, Budde RPJ, Bremerich J, Dacher JN, Loewe C, Wolf
F, et al. CT and MR imaging prior to transcatheter aortic valve implantation: standardisation of scanning protocols, measurements and reporting-a consensus document by the European Society of Cardiovascular Radiology (ESCR) [published correction appears in Eur Radiol 2020 Mar 2]. Eur Radiol 2020;30:2627-50. http://dx.doi. org/10.1007/s00330-019-06357-8

9. Tarakji KG, Patel D, Krishnaswamy A, Hussein A, Saliba WI, Navia JL, et al. Delayed Need For Permanent Pacemaker Implantation After TAVR Hospital Discharge Insights From The Brady-TAVR Study. J Am Coll Cardiol 2020, 75 (11 Supplement 1) 486.

10. Meduri CU, Kereiakes DJ, Rajagopal V, Makkar RR, O'Hair D, Linke A, et al. Pacemaker Implantation and Dependency After Transcatheter Aortic Valve Replacement in the REPRISE III Trial. J Am Heart Assoc 2019;8:e012594. http://dx.doi.org/10.1161/ JAHA.119.012594

11. von Elm E, Altman DG, Egger M, Pocock SJ, Gøtzsche PC, Vandenbroucke JP; STROBE Initiative. The Strengthening the Reporting of Observational Studies in Epidemiology (STROBE) statement: guidelines for reporting observational studies. Lancet 2007;370:14537. http://dx.doi.org/10.1016/S0140-6736(07)61602-X 